\title{
A promising two-dimensional channel material: monolayer antimonide phosphorus
}

\author{
Bo Cai $^{1 \dagger}$, Meiqiu Xie ${ }^{1 \dagger}$, Shengli Zhang ${ }^{1}$, Chengxi Huang ${ }^{2}$, Erjun $\mathrm{Kan}^{2}$, Xianping $\mathrm{Chen}^{3}$, Yu Gu ${ }^{1}$ \\ and Haibo Zeng ${ }^{*}$
}

\begin{abstract}
As the base of modern electronic industry, field-effect transistor (FET) requires the channel material to have both moderate bandgap and high mobility. The recent progresses indicate that few-layer black phosphorus has suitable bandgap and higher mobility than two-dimensional (2D) $\mathrm{MoS}_{2}$, but the experimentally achieved maximal mobility $\left(1000 \mathrm{~cm}^{2} \mathrm{~V}^{-1} \mathrm{~s}^{-1}\right)$ is still obviously lower than those of classical semiconductors $\left(1,400\right.$ and $5,400 \mathrm{~cm}^{2} \mathrm{~V}^{-1} \mathrm{~s}^{-1}$ for $\mathrm{Si}$ and InP). Here, for the first time, we report on monolayer antimonide phosphorus (SbP) as a promising $2 \mathrm{D}$ channel material with suitable direct bandgap, which can satisfy the on/off ratio, and with mobility as high as $10^{4} \mathrm{~cm}^{2} \mathrm{~V}^{-1} \mathrm{~s}^{-1}$ based on density functional theory calculation. In particular, $\alpha-S b_{1-x} P_{x}$ monolayers possess 0.3-1.6 eV bandgaps when $0.1 \leq x \leq 1$, which are greater than the minimum bandgap $(0.4 \mathrm{eV})$ required for large on/off ratio of FET. Surprisingly, the carrier mobilities of $\alpha-S b_{1-x} P_{x}$ monolayers exhibit very high upper limit approaching $2 \times 10^{4} \mathrm{~cm}^{2} \mathrm{~V}^{-1} \mathrm{~s}^{-1}$ when $0 \leq$ $x \leq 0.25$ due to the ultra-small effective mass of holes and electrons. This work reveals that $2 \mathrm{D} \mathrm{SbP}$ with both suitable bandgap and high mobility could be a promising candidate as eco-friendly high-performance FET channel materials avoiding short-channel effect in the post-silicon era, especially when considering the recent experimental success in realizing arsenide phosphorus (AsP) with similar structure.
\end{abstract}

Keywords: phosphorene, mobility, bandgap, density functional calculations, alloying strategy

\section{INTRODUCTION}

According to Moore's law, the chip performance will keep improving with continuing decrease of field-effect transistor (FET) size and increase of FET density. However, such trend has encountered a serious obstacle, so-called "shortchannel effect", which could make transistor nullify when the channel length is shorter than $10 \mathrm{~nm}$. The characteristic channel length $(\lambda)$ is related to the thickness $(t)$ and the dielectric constant $(\varepsilon)$ of semiconductor channel materials and the oxide as the following Equation (1) [1]:

$$
\lambda^{2}=\varepsilon_{\mathrm{s}} t_{\mathrm{s}} t_{\mathrm{OX}} / \varepsilon_{\mathrm{OX}} \text {. }
$$

Hence, adopting 2D semiconductors with ultrathin thickness $t_{\mathrm{s}}$ is a promising way to overcome "short-channel effect".

Besides the superiority from ultrathin structure, in order to be qualified as channel materials, the $2 \mathrm{D}$ semiconductors must also have a moderate band gap and high carrier mobility [2-5]. Graphene has mobility as high as $10^{5}$ $\mathrm{cm}^{2} \mathrm{~V}^{-1} \mathrm{~s}^{-1}$, but it is gapless, so on/off ratio of FET with graphene as channel is always low although many modifications such as narrowing into nanoribbons and nitrogen doping have been tried [3,6-15]. Subsequently, monolayer $\mathrm{MoS}_{2}$ exhibits significant indirect-to-direct band structure transition [16], but its highest mobility is as low as 200-500 $\mathrm{cm}^{2} \mathrm{~V}^{-1} \mathrm{~s}^{-1}[4,17,18]$, which is much lower than that of classical semiconductors such as silicon $\left(1,400 \mathrm{~cm}^{2} \mathrm{~V}^{-1} \mathrm{~s}^{-1}\right)$, and InP $\left(5,400 \mathrm{~cm}^{2} \mathrm{~V}^{-1} \mathrm{~s}^{-1}\right)$. Recently, black phosphorus (BP) was found to have both more suitable bandgap and higher mobility $\left(1000 \mathrm{~cm}^{2} \mathrm{~V}^{-1} \mathrm{~s}^{-1}\right)$ than monolayer $\mathrm{MoS}_{2}$, showing promising potentials as high-performance $2 \mathrm{D}$ channel material [2,19-25].

However, for practical applications of BP in FET, there are still some issues to be dealt with such as air-stability and mobility. For air-stability, despite the fact that BP is

\footnotetext{
${ }^{1}$ Institute of Optoelectronics \& Nanomaterials, Jiangsu Key Laboratory of Advanced Micro \& Nano Materials and Technology, College of Material Science and Engineering, Nanjing University of Science and Technology, Nanjing 210094, China

${ }^{2}$ Department of Applied Physics, Nanjing University of Science and Technology, Nanjing 210094, China

${ }^{3}$ College of Optoelectronic Engineering, Chongqing University, Chongqing 400044, China

T These authors contributed equally to this work.

*Corresponding author (email: zeng.haibo@njust.edu.cn)
} 
the most stable phase among the allotropes of phosphorus through theoretical calculation [26,27], it is found that $\mathrm{BP}$ can absorb moisture upon exposure to air without protection [28-30], and a hydrophobic polymer capping layer is typically required to provide improved air-stability for more than several weeks without degeneration of material properties [28]. Meanwhile, although the mobility of BP meets the requirement for FET applications, it remains lower than that of silicon $\left(1,400 \mathrm{~cm}^{2} \mathrm{~V}^{-1} \mathrm{~s}^{-1}\right), \operatorname{AlGaN}(2,000$ $\left.\mathrm{cm}^{2} \mathrm{~V}^{-1} \mathrm{~s}^{-1}\right)$, and $\operatorname{InP}\left(5,400 \mathrm{~cm}^{2} \mathrm{~V}^{-1} \mathrm{~s}^{-1}\right)$. Last year, $\boldsymbol{\alpha}-\mathrm{As}_{1-x} \mathrm{P}_{x}$ $(0.17<x<1)$ was fabricated adopting alloying strategy [31], even though the $\alpha-A s_{1-x} \mathrm{P}_{x}$ is theoretically predicted to transit from $\alpha$ to $\beta$ phase at $x=0.93$ [32]. Thus, tuning the carrier mobility of phosphorene is feasible through general alloying strategy experimentally. Given the biotoxicity of $\mathrm{As}_{1-x} \mathrm{P}_{x}$ (due to the presence of As) and the theoretical prediction that monolayer antimonene probably has a higher air stability than phosphorene and $\alpha-\mathrm{As}_{1-x} \mathrm{P}_{x}$ [33], we employ the $\mathrm{Sb}$ element to modulate the electronic properties of $\alpha$-phosphorene.

Here, we report on the design of monolayer antimonide phosphorus ( $\mathrm{SbP}$ ) as a promising 2D channel material with suitable bandgap and mobility as high as $10^{4} \mathrm{~cm}^{2} \mathrm{~V}^{-1} \mathrm{~s}^{-1}$ based on density functional theory (DFT). According to the calculated results, we found that dynamically stable monolayer $\alpha-\mathrm{Sb}_{1-x} \mathrm{P}_{x}(0.1 \leq x \leq 0.25)$ would have an overt direct bandgap and a mobility one order of magnitude higher than phosphorene. 2D SbP tends to stabilize itself in the form of a strongly folded hexagonal honeycomb sheet with 'troughs' along the zigzag direction, which is confirmed by the corresponding phonon dispersions without any imaginary frequency. Notably, 2D SbP possesses $0.3-1.6 \mathrm{eV}$ bandgap when $0.1 \leq x \leq 1$, which would be larger than the basic requirement $0.4 \mathrm{eV}$ for large on/off ratio of FET. Surprisingly, the carrier mobility of $2 \mathrm{D}$ SbP exhibits high upper limit approaching $2 \times 10^{4} \mathrm{~cm}^{2} \mathrm{~V}^{-1} \mathrm{~s}^{-1}$ when $0 \leq x \leq 0.25$. The underlying origins are attributed to the ultralight hole and electron due to the strong dispersion of valence band maximum (VBM) and conduction band minimum (CBM). This work reveals that 2D SbP could be of both suitable bandgap and high mobility, and hence a promising candidate as eco-friendly high-performance FET channel material avoiding short-channel effect.

\section{COMPUTATIONAL DETAILS}

All the DFT calculations were performed by using the Vienna ab simulation package (VASP) [34], under periodic boundary conditions. The projector-augmented-wave (PAW) potential with $\mathrm{P}$ and $\mathrm{Sb}$ was described as valence.
Spin-orbital coupling (SOC) effect was introduced to treat electrons of antimony element. Geometry optimization and band structure calculations were carried out under the Perdew, Burke, and Ernzerholf (PBE) exchange-correlation functional [35] and band structures were checked by the Heyd-Scuseria-Ernzerhof hybrid functional (HSE06) [36]. A kinetic energy cutoff of $500 \mathrm{eV}$ was set on a grid of $21 \times 21 \times 1 \mathrm{k}$-points for $\alpha-\mathrm{Sb}_{1-x} \mathrm{P}_{x}$ monolayer for PBE. And the kinetic energy cutoff of $500 \mathrm{eV}$ and a grid of $5 \times 5 \times 1$ k-points were set for HSE06. A sufficiently large $20 \AA$ vacuum region was used to isolate the two-dimensional (2D) structures to rule out the neighboring interaction along the $c$-axis. Phonon dispersion calculations were performed using Cambridge Sequential Total Energy Package (CASTEP) [37]. The parameter reliability can be verified by the optimized $\alpha$-phosphorene lattice parameters $a=$ $4.62 \AA$ and $b=3.30 \AA$ ( $a=4.58 \AA, b=3.32 \AA$ in ref. [38]).

In a $2 \mathrm{D}$ system, the carrier mobility is given by the equation:

$$
\mu_{2 \mathrm{D}}=\frac{e \hbar^{3} C_{2 \mathrm{D}}}{k_{\mathrm{B}} T m_{\mathrm{e}}{ }^{*} m_{\mathrm{d}}\left(E_{1}^{i}\right)^{2}},
$$

where $C_{2 \mathrm{D}}$ is the elastic modulus of the longitudinal strain in the propagation directions (both armchair and zigzag) of the longitudinal acoustic wave. $C_{2 \mathrm{D}}$ is derived from

$$
C_{2 \mathrm{D}}=\frac{1}{S_{0}} \frac{\partial^{2} E}{\partial \delta^{2}},
$$

where $S_{0}$ is the lattice volume at equilibrium for a $2 \mathrm{D}$ system. $E$ is the total energy and $\delta$ is the applied uniaxial strain $\Delta l / l_{0}$. The parameter $m_{\mathrm{e}}{ }^{*}$ is the effective mass in the transport direction and $m_{\mathrm{d}}$ is the average effective mass determined by

$$
m_{\mathrm{d}}=\sqrt{m_{x}^{*} m_{y}^{*}} .
$$

The deformation potential $E_{1}{ }^{i}$ is defined as

$$
E_{1}^{i}=\Delta V_{i} / \delta,
$$

where $\Delta V_{i}$ is the energy change for the $i$-th band with lattice dilation $\delta[38-40]$.

\section{RESULTS}

\section{Antimonide phosphorene structures}

There are many phosphorus allotropes in previous theory predicts [41-43]. However, only a-phosphorene has been successfully synthesized $[2,21,44-46]$ despite the fact that $\beta-A s_{1-x} \mathrm{P}_{x}$ has lower energy than $\alpha$ phase according to simulations [31,42]. So, we only explored the atomic structure 
of $\alpha-\mathrm{Sb}_{1-x} \mathrm{P}_{x}$ monolayer in the composition $0 \leq x \leq 1$. In particular, we considered different composition of $\mathrm{Sb}$ and $\mathrm{P}$ atoms with $x=0,0.25,0.5,0.75$ and 1 , as shown in Figs S1-4 (Supplementary materials). Structural model of $\mathrm{Sb}_{0.75} \mathrm{P}_{0.25}$, which has the best performance among the five models in our work, is shown in Fig. 1. And the $\mathrm{Sb}_{0.75} \mathrm{P}_{0.25}$ model here is the lowest energy model among twenty-eight different $\mathrm{Sb}_{0.75} \mathrm{P}_{0.25}$ models, as shown in Fig. S5 and Table S1. The relaxed $\alpha-\mathrm{Sb}_{0.75} \mathrm{P}_{0.25}$ structure has a $2 \mathrm{D}$ rectangular lattice with $a=4.85 \AA$ and $b=4.08 \AA$ and contains one $\mathrm{P}$ atom and three $\mathrm{Sb}$ atoms in the primitive unit cell. The monolayer $\mathrm{Sb}_{0.75} \mathrm{P}_{0.25}$ consists of two unparallel atomic planes unlike phosphorene. It is due to the fact that during the substitution of $\mathrm{Sb}$ atoms for $\mathrm{P}$ atoms in pristine phosphorene, the $\mathrm{Sb}$ atoms slightly displace outwards and the lattice constants of the rectangular primitive cell are raised in order to increase the stability. As Sb concentration increased to $100 \%$, the $\mathrm{Sb}_{1-x} \mathrm{P}_{x}$ monolayer recovers two parallel atomic planes and the lattice constants increase to $a=4.91 \AA$ and $b=4.35 \AA$ from $a=4.62 \AA$ and $b=3.30 \AA$ for phosphorene (lattice constants are given in Table S2 for further references). For $\mathrm{Sb}_{0.5} \mathrm{P}_{0.5}$, the energy of model only containing $\mathrm{Sb}-\mathrm{P}$ bond is lower than that of which contains $\mathrm{Sb}-\mathrm{Sb}$, $\mathrm{P}-\mathrm{P}$, and $\mathrm{Sb}-\mathrm{P}$ bonds, as shown in Supplementary materials. Hence, we took the model which only contains Sb-P bond to calculate the phonon dispersion and band structure.

\section{Stability and experimental feasibility}

Stability and experimental feasibility have to be considered for designing 2D materials through computation. As shown in Fig. 2, no soft phonon modes are available in the computed phonon dispersion spectra of $\alpha-\mathrm{Sb}_{1-x} \mathrm{P}_{x}(x$ $=0,0.25,0.5$ and 0.75$)$ monolayer, which demonstrates their experimental feasibility. Soften dispersion in $S$ point in $\mathrm{Sb}_{0.75} \mathrm{P}_{0.25}$ and $\mathrm{Sb}_{1} \mathrm{P}_{0}$ indicates that these materials may have negative group velocity, which is a rare property. And a more linear than quadratic dispersion at $\Gamma$ point in $\mathrm{Sb}_{0.75} \mathrm{P}_{0.25}$ and $\mathrm{Sb}_{1} \mathrm{P}_{0}$ indicates that ZA modes couple with LA and TA modes to some extent. On the other hand, a different structure of $\alpha$-antimonene (we name it $\alpha^{\prime}$-antimonene for the moment, see Fig. S6) has been proved to be a stable structure several months ago $[47,48]$. The structure of $\alpha^{\prime}$-antimonene is similar to $\mathrm{Sb}_{0.5} \mathrm{P}_{0.5}$-one of two $\mathrm{Sb}$ atoms is slightly located outwards in either plane of the primitive unit cell. In fact, the total energy of $\alpha$-antimonene is $4.7 \mathrm{meV} /$ atom lower than that of $\alpha^{\prime}$-antimonene in our work. Detailed analysis can be seen in Supplementary materials. To further characterize the stability of $\mathrm{Sb}_{0.75}$
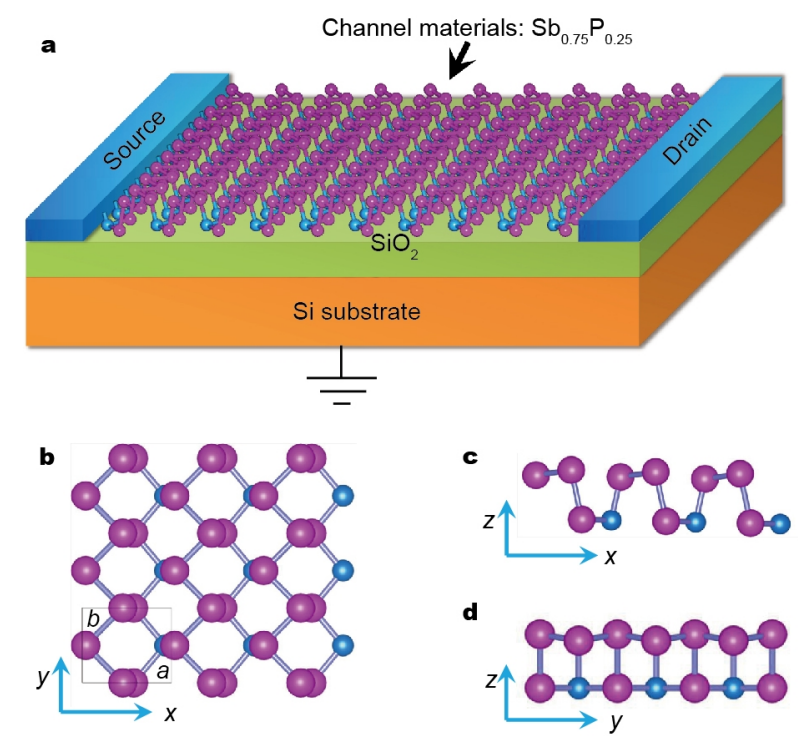

Figure 1 (a) Schematic of a monolayer antimonide phosphorus FET. (b) Top view and $(c, d)$ two side views of relaxed $\mathrm{Sb}_{0.75} \mathrm{P}_{0.25}$, which has the best performance.

$\mathrm{P}_{0.25}$, the formation energy $\left(E^{\mathrm{f}}\right)$ was also calculated. The $E^{f}$ of monolayer $\mathrm{Sb}_{0.75} \mathrm{P}_{0.25}$ is defined as $E^{\mathrm{f}}=E^{\mathrm{tot}}\left(\mathrm{Sb}_{0.75} \mathrm{P}_{0.25}\right)-E^{\mathrm{tot}}($ antimonene $)-\mu_{\mathrm{P}}+\mu_{\mathrm{sb}}$, where $E^{\text {tot }}\left(\mathrm{Sb}_{0.75} \mathrm{P}_{0.25}\right)$ and $E^{\text {tot }}($ antimonene) are the total energy derived from a unit cell calculation for $\mathrm{Sb}_{0.75} \mathrm{P}_{0.25}$ and $\alpha$-antimonene, respectively, $\mu_{\mathrm{P}}$ and $\mu_{\mathrm{sb}}$ are the phosphorus and antimony chemical potential, respectively. According to the calculation, the $E^{f}$ is $-1.02 \mathrm{eV}$. The phonon dispersion and formation energy indicate that monolayer $\mathrm{Sb}_{0.75} \mathrm{P}_{0.25}$ is stable and should be achievable in experiments. Moreover, we investigated the Gibbs energy of formation $\Delta G_{\mathrm{f}}$ to evaluate whether phase separation will happen in bulk system. The $\Delta G_{\mathrm{f}}$ is defined as $\Delta G_{\mathrm{f}}$ $=G\left(\mathrm{Sb}_{1-x} \mathrm{P}_{x}\right)-x \mathrm{G}(\mathrm{P})-(1-x) G(\mathrm{Sb})$, where $G=E-T S$ and entropy $S=-k_{\mathrm{B}}[x \log x+(1-x) \log (1-x)]$. It is worth noting that phase separation is spontaneous in bulk $\mathrm{Sb}_{1-x} \mathrm{P}_{x}$ in low concentration of $\mathrm{Sb}$ at $300 \mathrm{~K}$, as shown in Fig. 2e. It can be explained as follows. When small quantities of $\mathrm{Sb}$ mixed with $\mathrm{P}, \mathrm{P}$ atoms are squeezed around $\mathrm{Sb}$ atoms, which induces a rapid energy increase according to Lennard-Jones potential [49]. However, when smaller radius $\mathrm{P}$ mixed with $\mathrm{Sb}, \mathrm{Sb}$ atoms are stretched around $\mathrm{P}$ atom, which only induces a slow energy increase. Thus, high concentration of $\mathrm{Sb}$ system is phase mixture, while low concentration system is phase separation.

How to manufacture the materials in the future experiments? Black arsenic-phosphorus $\left(\alpha-A s_{1-x} \mathrm{P}_{x}\right.$, with $x$ in the range of $0.17-1$ ) was recently manufactured via a synthetic 

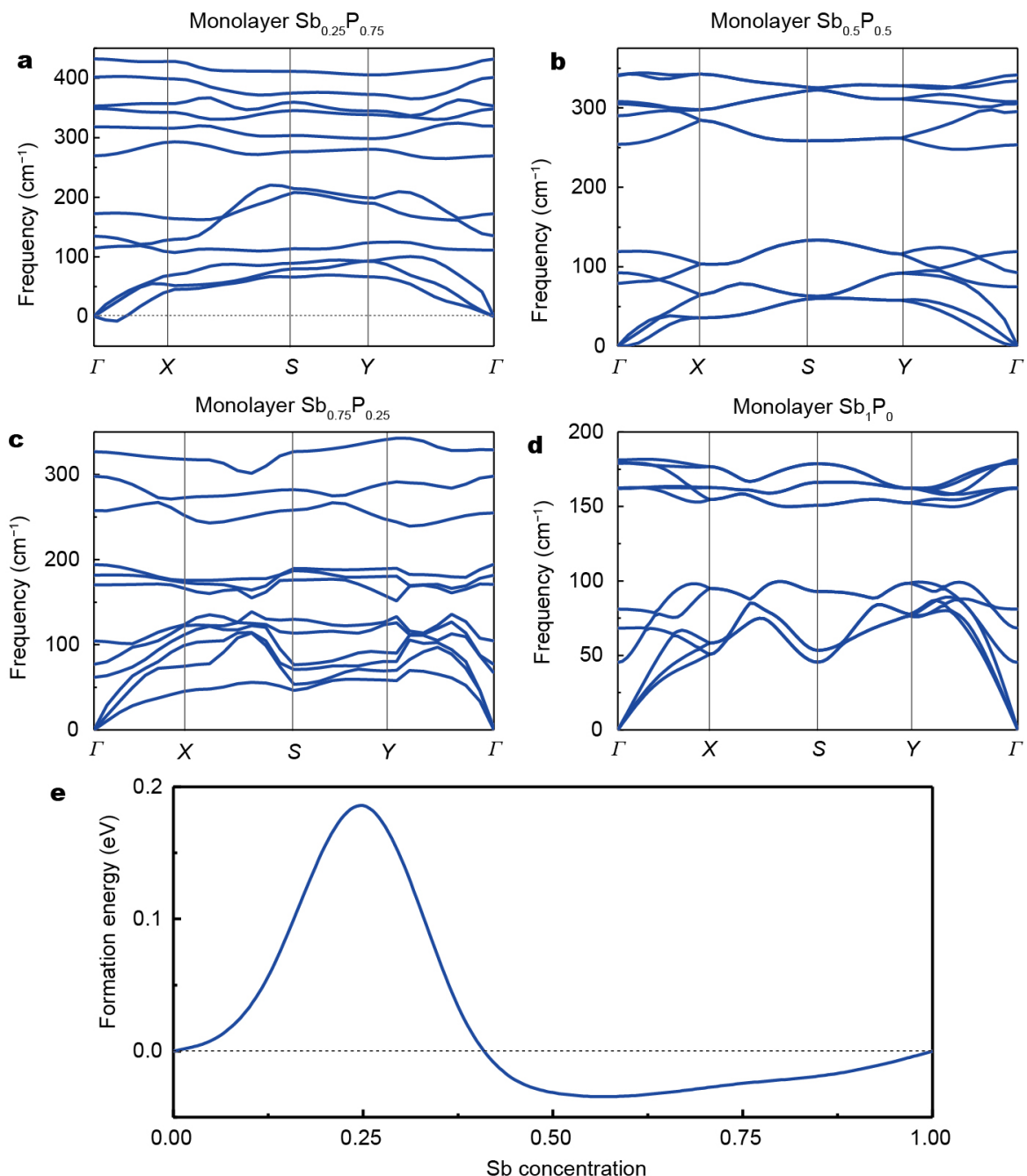

Figure 2 Phonon dispersions of monolayer (a) $\mathrm{Sb}_{0.25} \mathrm{P}_{0.75}$, (b) $\mathrm{Sb}_{0.5} \mathrm{P}_{0.5}$, (c) $\mathrm{Sb}_{0.75} \mathrm{P}_{0.25}$ and (d) $\mathrm{Sb}_{1} \mathrm{P}_{0}$, respectively. The phonon dispersions show the stability of these models. (e) Formation Gibbs energy of bulk $\mathrm{Sb}_{1-x} \mathrm{P}_{x}$ at $300 \mathrm{~K}$, indicating phase separation will happen in low concentration of $\mathrm{Sb}$.

approach adopting the alloying strategy [31]. The experimental protocols are expected to provide insights for fabrication of $\mathrm{Sb}_{1-x} \mathrm{P}_{x}$. The synthesis of $\alpha-\mathrm{Sb}_{1-x} \mathrm{P}_{x}$ samples should be feasible adopting the similar vapor transport method by tuning the ratio of red phosphorus and metallic antimony since the structure of $\mathrm{Sb}_{1-x} \mathrm{P}_{x}$ and metallic antimony are the same to $A s_{1-x} \mathrm{P}_{x}$ and gray arsenic, respectively. After synthesis, the bulk samples can be mechanically exfoliated by tapes.

\section{Electronic properties}

As expected, the introduction of $\mathrm{Sb}$ into a-phosphorene will affect the electronical structures as shown in Fig. 3. Pure a-phosphorene (Fig. 3a) is a typical semiconductor with a direct bandgap of $0.9 \mathrm{eV}$ at $\Gamma$ within PBE functional. $\mathrm{A}$ and $\mathrm{B}$ are labels of valence band $(\mathrm{VB})$ and conduction band $(\mathrm{CB})$ states, respectively. As $x$ value increasing to 1 , the VBM and CBM shift to $X_{1}(0.38,0,0)$ (labeled as $C$ and D) with a bandgap of $0.05 \mathrm{eV}$ with SOC effect in the band structure of $\alpha$-antimonene within PBE exchange-correlation functional, as shown in Fig. 3e. It appears to be a topological insulator. Unfortunately, it is a normal insulator, which was confirmed via topological invariant Z2 [50,51]. Examined by HSE06 hybrid functional, the bandgaps are corrected to $1.6 \mathrm{eV}$ for phosphorene and $0.3 \mathrm{eV}$ for antimonene. Fig. $3 b-d$ show the band structure of $S b_{1-x} \mathrm{P}_{x}$ with $x=0.75,0.5$ and 0.25 , respectively. The three figures can provide some information about the variation of band structure of $\mathrm{Sb}_{1-x} \mathrm{P}_{x}(0 \leq x \leq 1)$. It is clear that the two VB states $\mathrm{A}$ and $\mathrm{C}$ dominate for the $\mathrm{VBM}$, while four $\mathrm{CB}$ states $\mathrm{B}, \mathrm{D}, \mathrm{E}$ and $\mathrm{F}$ compete for the CBM.

The variation tendency of $\mathrm{VB}$ states $\mathrm{A}, \mathrm{C}$ and $\mathrm{CB}$ states 

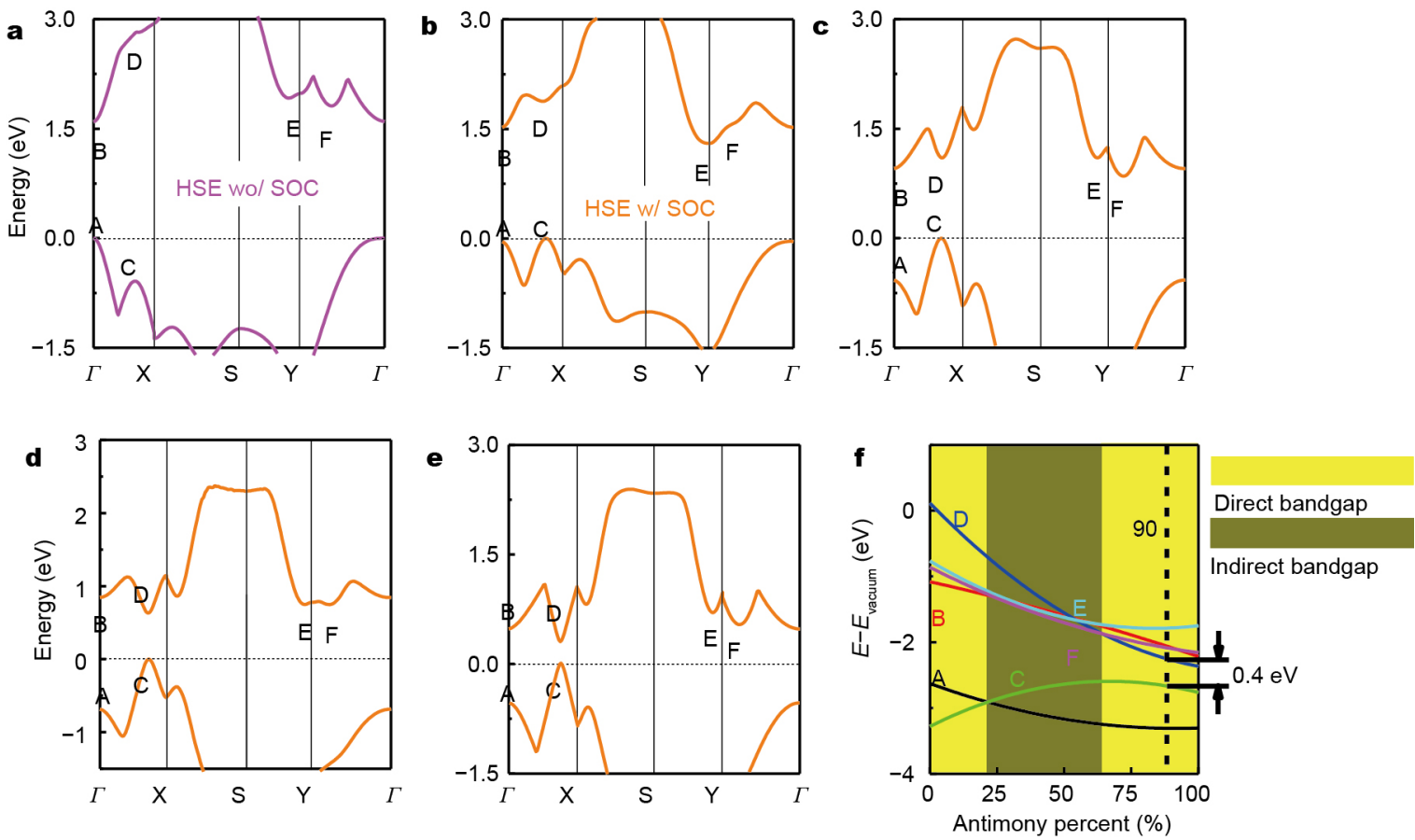

Figure 3 VBM and CBM of (a) $\mathrm{Sb}_{0} \mathrm{P}_{1}$ ( $\alpha$-phosphorene), (b) $\mathrm{Sb}_{0.25} \mathrm{P}_{0.75}$, (c) $\mathrm{Sb}_{0.5} \mathrm{P}_{0.5}$, (d) $\mathrm{Sb}_{0.75} \mathrm{P}_{0.25}$ and (e) $\mathrm{Sb}_{1} \mathrm{P}_{0}$ ( $\alpha$-antimonene) monolayers, respectively. Phosphorene band structure is under HSE functional without SOC effect. Other systems are investigated via employing HSE functional with SOC effect. (f) The variation tendency of valence band state A, C and conduction band state B, D, E, F. The yellow area represents that it is direct bandgap semiconductor in this proportion range, and dark yellow area is indirect bandgap.

B, D, E, $\mathrm{F}$ are given in Fig. 3f. The competition for VBM and CBM has three stages. (i) When $0.79 \leq x \leq 1, \mathrm{VB}$ states $A$ and $C B$ states $B$ rule the VBM and CBM, respectively. In this case, $\mathrm{Sb}_{1-x} \mathrm{P}_{x}$ monolayer is a direct bandgap semiconductor. (ii) When $0.36 \leq x \leq 0.79$, VB states $\mathrm{C}$ replaces the status of $A$, and becomes new VBM. And CBM location changes from $\mathrm{CB}$ states $\mathrm{B}$ to $\mathrm{E}$ to $\mathrm{F}$. In this case, $\mathrm{Sb}_{1-x} \mathrm{P}_{x}$ monolayer is an indirect bandgap semiconductor. (iii) When $0 \leq x \leq 0.36, \mathrm{CB}$ states $\mathrm{D}$ overthrows the rule of $\mathrm{B}, \mathrm{E}$ and $\mathrm{F}$, and governs $\mathrm{CBM}$. Meanwhile, $\mathrm{C}$ is still VBM. In this case, $\mathrm{Sb}_{1-x} \mathrm{P}_{x}$ monolayer becomes direct bandgap semiconductor again. The band gap ranges from 0.30 to 0.63 $\mathrm{eV}$. And at the range from $\mathrm{Sb}_{0.64} \mathrm{P}_{0.36}$ to $\mathrm{Sb}_{0.9} \mathrm{P}_{0.1}$, the direct band gap is larger than $0.4 \mathrm{eV}$, meeting the requirement of on/off ratio. At the $\mathrm{VB}$ state $\mathrm{C}$ and $\mathrm{CB}$ state $\mathrm{D}$, the bandgap wave vector disperses more strongly than $A$ and $B$, indicating a smaller carrier effective mass according to $1 / \mathrm{m}^{*}=$ $\left(\partial^{2} E / \partial k^{2}\right) / \hbar^{2}$, where $m^{*}$ is the carrier effective mass. According to the equation, the effective masses are quite small, namely $0.04 m_{0}$ for electron and hole in $\mathrm{Sb}_{0.75} \mathrm{P}_{0.25}$ and $0.01 m_{0}$ for electron and hole in antimonene along the armchair direction, indicating the material may have ultrahigh mobility in $\mathrm{Sb}_{0.75} \mathrm{P}_{0.25}$ and $\mathrm{Sb}_{1} \mathrm{P}_{0}$.

We can infer the carrier mobility, which is strongly influenced by their effective masses, through the electronic properties of $\mathrm{Sb}_{1-x} \mathrm{P}_{x}$ monolayer. Here, we used a phonon-limit scattering model, in which the primary mechanism limiting carrier mobility is scatting because of phonons $[38,52]$. Besides effective mass, the deformation potential $E_{1}$ and the elastic modulus $C_{2 \mathrm{D}}$ in the transmission direction of the longitudinal acoustic wave are the most related factors affecting mobility $[39,53]$. The predicted mobilities for the five $\mathrm{Sb}_{1-x} \mathrm{P}_{x}$ monolayer systems are shown in Fig. 4. The electron mobilities along the armchair and zigzag direction in phosphorene are 3,540 and $104 \mathrm{~cm}^{2} \mathrm{~V}^{-1} \mathrm{~s}^{-1}$, respectively. And the hole mobilities along armchair and zigzag direction in phosphorene are 1,346 and $4,190 \mathrm{~cm}^{2} \mathrm{~V}^{-1} \mathrm{~s}^{-1}$, respectively. Both the mobilities of electron and hole along the armchair direction are higher than the zigzag direction because the band structure of phosphorene is more dispersive along the armchair direction. In $\mathrm{Sb}_{0.75} \mathrm{P}_{0.25}$ and antimonene, the $\mathrm{VB}$ and $\mathrm{CB}$ disperse rather strongly along the armchair direction, resulting in ultralight electrons and holes. Both the value of $m_{\mathrm{e}}{ }^{*}$ and $m_{\mathrm{h}}{ }^{*}$ along the armchair direction are very striking in that it is several orders of magnitude smaller than typical values of $m^{*}$, which are $9.52 m_{0}$ for holes of $\mathrm{Sb}_{0} \mathrm{P}_{1}$ along zigzag direction, $1.07 m_{0}$ for electrons of $\mathrm{Sb}_{0} \mathrm{P}_{1}$ along the armchair 

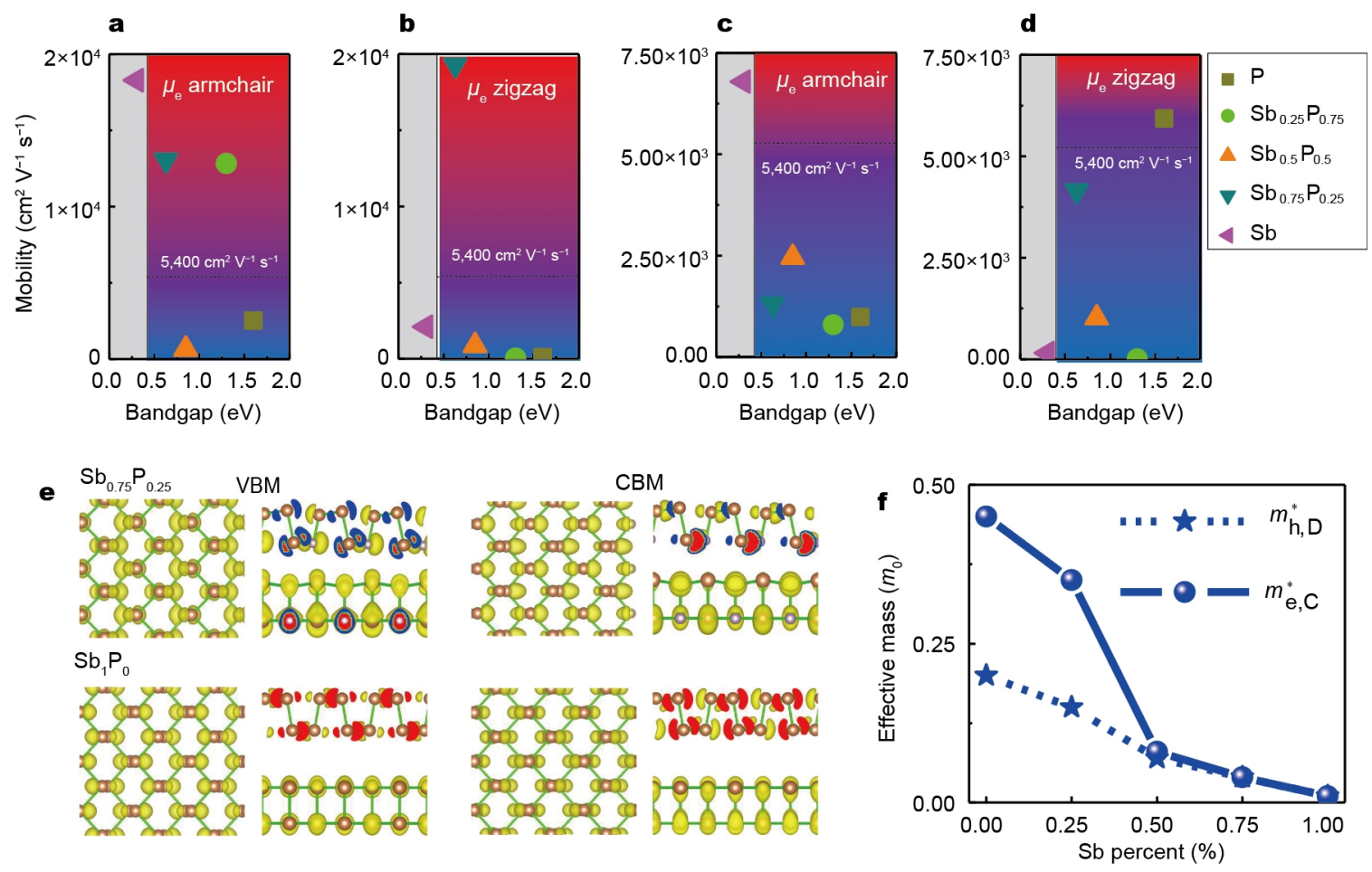

Figure 4 (a-d) Band gaps and mobilities of $\mathrm{Sb}_{1-x} \mathrm{P}_{x}$ monolayers. The band gap is smaller than $0.4 \mathrm{eV}$ in gray area. (e) $\mathrm{Spatial}$ structure of wavefunctions, which is the influencing factor of deformation potential, for $\mathrm{Sb}_{0.75} \mathrm{P}_{0.25}$ and antimonene, respectively. (f) Effective masses for $\mathrm{VB}$ state $\mathrm{C}$ and $\mathrm{CB}$ state $\mathrm{D}$ along the armchair direction.

direction, $4.64 m_{0}$ for holes of $S_{0.25} \mathrm{P}_{0.75}$ along the zigzag direction, $3.42 m_{0}$ for electrons of $\mathrm{Sb}_{0.25} \mathrm{P}_{0.75}$ along the armchair direction, etc. While the deformation potentials $E_{1}$ are much higher than that of phosphorene and the values of elastic moduli $C_{2 \mathrm{D}}$ are about $60 \%$ of phosphorene. As a result, the mobility of $S b_{0.75} \mathrm{P}_{0.25}$ and antimonene is $2.5-4$ times higher than that of phosphorene, that is, 11,200 and $18,500 \mathrm{~cm}^{2} \mathrm{~V}^{-1} \mathrm{~s}^{-1}$ compared with $4,190 \mathrm{~cm}^{2} \mathrm{~V}^{-1} \mathrm{~s}^{-1}$, respectively. More details can be seen in Table S3. The difference between our result and that of Ref. [32] is caused by different calculation method.

Does $\mathrm{Sb}_{1-x} \mathrm{P}_{x}(0 \leq x \leq 0.25)$ monolayer have high mobility, similar to $S b_{0.75} \mathrm{P}_{0.25}$ and antimonene? The origin of high mobility should be found out before we answer this question. Fig. $4 \mathrm{~b}$ shows the spatial structure of VBM wavefunctions of two models $\left(\mathrm{Sb}_{0.75} \mathrm{P}_{0.25}\right.$, and antimonene). In $\mathrm{Sb}_{0.75} \mathrm{P}_{0.25}$ and antimonene systems, VBM (VB state C) and CBM (CB state D) are mixture of $p_{x}$ and $s$ orbitals, which is different from the $p_{z}$ orbital in phosphorene. The different spatial structure introduces a different anisotropic behavior between phosphorene and high Sb concentration monolayer $\mathrm{Sb}_{1-x} \mathrm{P}_{x}$. Since the structural deformations due to longitudinal acoustic phonon are represented as homogeneous, symmetrical gradual change [38,52], the value of
$E_{1}$ in $\mathrm{Sb}_{1-x} \mathrm{P}_{x}(0 \leq x \leq 0.25)$ monolayer should be located between the value of $E_{1}$ in $S b_{0.75} \mathrm{P}_{0.25}$ and antimonene as long as the bond natures of CBM and VBM of $S_{0.75} \mathrm{P}_{0.25}$ and antimonene remain unchanged. Meanwhile, the high mobility relies on the ultralight electrons and holes along the armchair direction primarily. And the value of effective masses, determined by state $\mathrm{D}$, decreases monotonically with the increase of $\mathrm{Sb}$ content, as shown in Fig. $4 c$. Thus, $\mathrm{Sb}_{1-x} \mathrm{P}_{x}(0$ $\leq x \leq 0.25)$ monolayers have small effective masses, resulting in high mobilities.

\section{CONCLUSIONS}

In summary, we predicted a new two-dimensional material $\mathrm{Sb}_{1-x} \mathrm{P}_{x}$ by first principle DFT calculation. $\mathrm{Sb}_{1-x} \mathrm{P}_{x}$ $(0 \leq x \leq 1)$ has a puckered honeycomb lattice similar to $a$-phosphorene. The phonon dispersion results indicate that $\mathrm{Sb}_{1-x} \mathrm{P}_{x}$ are achievable in experiments. The direct bandgap of $\mathrm{Sb}_{1-x} \mathrm{P}_{x}(0.1 \leq x \leq 0.25)$ are in the range of 0.4 to $0.63 \mathrm{eV}$, which can meet the need of on/off ratio. Moreover, the band structure disperses strongly along the armchair direction, resulting in ultralight electrons and holes between $\mathrm{Sb}_{0.75} \mathrm{P}_{0.25}$ and antimonene. The carrier mobilities, which are strongly influenced by their ultra-small effective masses, are rather high. The values can reach 
3-5 times higher than that of phosphorene. Therefore, the mobilities may surpass $\mathrm{Si}$, and InP. Our study of $\mathrm{Sb}_{1-x} \mathrm{P}_{x}$ may provide a new material that has potential applications in microelectronics and optoelectronics devices.

\section{Received 6 July 2016; accepted 6 August 2016;}

published online 18 August 2016

1 Liu H, Neal AT, Ye PD. Channel length scaling of $\mathrm{MoS}_{2}$ MOSFETs. ACS Nano, 2012, 6: 8563-8569

2 Li L, Yu Y, Ye GJ, et al. Black phosphorus field-effect transistors. Nat Nanotech, 2014, 9: 372-377

3 Schwierz F. Graphene transistors. Nat Nanotech, 2010, 5: 487-496

4 Radisavljevic B, Radenovic A, Brivio J, et al. Single-layer $\mathrm{MoS}_{2}$ transistors. Nat Nanotech, 2011, 6: 147-150

5 Yoon Y, Ganapathi K, Salahuddin S. How good can monolayer $\mathrm{MoS}_{2}$ transistors be? Nano Lett, 2011, 11: 3768-3773

6 Novoselov KS, Geim AK, Morozov SV, et al. Two-dimensional gas of massless Dirac fermions in graphene. Nature, 2005, 438: $197-200$

7 Geim AK, Novoselov KS. The rise of graphene. Nat Mater, 2007, 6: 183-191

8 Hwang EH, Das Sarma S. Acoustic phonon scattering limited carrier mobility in two-dimensional extrinsic graphene. Phys Rev B, 2008, 77: 115449

9 Cai B, Zhang S, Yan Z, et al. Noncovalent molecular doping of two-dimensional materials. ChemNanoMat, 2015, 1: 542-557

10 Fiori G, Iannaccone G. Simulation of graphene nanoribbon fieldeffect transistors. IEEE Electron Device Lett, 2007, 28: 760-762

11 Tang Q, Zhou Z. Graphene-analogous low-dimensional materials. Prog Mater Sci, 2013, 58: 1244-1315

12 Tang Q, Zhou Z, Chen Z. Graphene-related nanomaterials: tuning properties by functionalization. Nanoscale, 2013, 5: 4541-4583

$13 \mathrm{Li} \mathrm{Y}$, Zhou Z, Shen $\mathrm{P}$, et al. Spin gapless semiconductor-metal-half-metal properties in nitrogen-doped zigzag graphene nanoribbons. ACS Nano, 2009, 3: 1952-1958

14 Li Y, Li F, Chen Z. Graphane/fluorographene bilayer: considerable $\mathrm{C}-\mathrm{H} \cdots \mathrm{F}-\mathrm{C}$ hydrogen bonding and effective band structure engineering. J Am Chem Soc, 2012, 134: 11269-11275

15 Zhang X, Zhang H, Lin Z, et al. Recent advances and challenges of stretchable supercapacitors based on carbon materials. Sci China Mater, 2016, 59: 475-494

16 Mak KF, Lee C, Hone J, et al. Atomically thin $\mathrm{MoS}_{2}$ : a new directgap semiconductor. Phys Rev Lett, 2010, 105: 136805

17 Schmidt H, Wang S, Chu L, et al. Transport properties of monolayer $\mathrm{MoS}_{2}$ grown by chemical vapor deposition. Nano Lett, 2014, 14: 1909-1913

18 Yang X, Li Q, Hu G, et al. Controlled synthesis of high-quality crystals of monolayer $\mathrm{MoS}_{2}$ for nanoelectronic device application. Sci China Mater, 2016, 59: 182-190

19 Fei R, Yang L. Strain-engineering the anisotropic electrical conductance of few-layer black phosphorus. Nano Lett, 2014, 14: 2884-2889

20 Liu H, Neal AT, Zhu Z, et al. Phosphorene: an unexplored 2D semiconductor with a high hole mobility. ACS Nano, 2014, 8: 4033-4041

21 Das S, Zhang W, Demarteau M, et al. Tunable transport gap in phosphorene. Nano Lett, 2014, 14: 5733-5739

22 Ong ZY, Zhang G, Zhang YW. Anisotropic charged impurity-limited carrier mobility in monolayer phosphorene. J Appl Phys, 2014, 116: 214505
23 Velizhanin KA, Shahbazyan TV. Exciton-plasmaritons in graphene/semiconductor structures. Phys Rev B, 2014, 90: 085403

24 Han X, Morgan Stewart H, Shevlin SA, et al. Strain and orientation modulated bandgaps and effective masses of phosphorene nanoribbons. Nano Lett, 2014, 14: 4607-4614

25 Dai J, Zeng XC. Bilayer phosphorene: effect of stacking order on bandgap and its potential applications in thin-film solar cells. J Phys Chem Lett, 2014, 5: 1289-1293

26 Zhang S, Xie M, Li F, et al. Semiconducting group 15 monolayers: a broad range of band gaps and high carrier mobilities. Angew Chem Int Ed, 2016, 55: 1666-1669

27 Zhao T, He CY, Ma SY, et al. A new phase of phosphorus: the missed tricycle type red phosphorene. J Phys-Condens Matter, 2015, 27: 265301

28 Kim JS, Liu Y, Zhu W, et al. Toward air-stable multilayer phosphorene thin-films and transistors. Sci Rep, 2015, 5: 8989

29 Ziletti A, Carvalho A, Campbell DK, et al. Oxygen defects in phosphorene. Phys Rev Lett, 2015, 114: 046801

30 Wang G, Pandey R, Karna SP. Phosphorene oxide: stability and electronic properties of a novel two-dimensional material. Nanoscale, 2015, 7: 524-531

31 Liu B, Köpf M, Abbas AN, et al. Black arsenic-phosphorus: layered anisotropic infrared semiconductors with highly tunable compositions and properties. Adv Mater, 2015, 27: 4423-4429

32 Zhu Z, Guan J, Tománek D. Structural transition in layered $\mathrm{As}_{1-x} \mathrm{P}_{x}$ compounds: a computational study. Nano Lett, 2015, 15: 6042-6046

33 Üzengi Aktürk O, Aktürk E, Ciraci S. Effects of adatoms and physisorbed molecules on the physical properties of antimonene. Phys Rev B, 2016, 93: 035450

34 Kresse G, Furthmüller J. Efficient iterative schemes for ab initio total-energy calculations using a plane-wave basis set. Phys Rev B, 1996, 54: 11169-11186

35 Perdew JP, Burke K, Ernzerhof M. Generalized gradient approximation made simple. Phys Rev Lett, 1996, 77: 3865-3868

36 Heyd J, Scuseria GE, Ernzerhof M. Erratum: "Hybrid functionals based on a screened Coulomb potential". [J Chem Phys, 118, 8207 (2003)]. J Chem Phys, 2006, 124: 219906

37 Clark SJ, Segall MD, Pickard CJ, et al. First principles methods using CASTEP. Zeitschrift für Kristallographie-Crystline Mater, 2005, 220: 567-570

38 Qiao J, Kong X, Hu ZX, et al. High-mobility transport anisotropy and linear dichroism in few-layer black phosphorus. Nat Commun, 2014, 5: 4475

39 Xi J, Long M, Tang L, et al. First-principles prediction of charge mobility in carbon and organic nanomaterials. Nanoscale, 2012, 4: 4348-4369

40 Cai Y, Zhang G, Zhang YW. Polarity-reversed robust carrier mobility in monolayer $\mathrm{MoS}_{2}$ nanoribbons. J Am Chem Soc, 2014, 136: 6269-6275

41 Wu M, Fu H, Zhou L, et al. Nine new phosphorene polymorphs with non-honeycomb structures: a much extended family. Nano Lett, 2015, 15: 3557-3562

42 Guan J, Zhu Z, Tománek D. Phase coexistence and metal-insulator transition in few-layer phosphorene: a computational study. Phys Rev Lett, 2014, 113: 046804

43 Guan J, Zhu Z, Tománek D. Tiling phosphorene. ACS Nano, 2014, 8: $12763-12768$

44 Wang $\mathrm{H}$, Wang $\mathrm{X}$, Xia F, et al. Black phosphorus radio-frequency transistors. Nano Lett, 2014, 14: 6424-6429

45 Liang L, Wang J, Lin W, et al. Electronic bandgap and edge reconstruction in phosphorene materials. Nano Lett, 2014, 14: 


\section{0-6406}

46 Zhang Z, Xin X, Yan Q, et al. Two-step heating synthesis of sub-3 millimeter-sized orthorhombic black phosphorus single crystal by chemical vapor transport reaction method. Sci China Mater, 2016, 59: $122-134$

47 Aktürk OÜ, Özçelik VO, Ciraci S. Single-layer crystalline phases of antimony: antimonenes. Phys Rev B, 2015, 91: 235446

48 Wang G, Pandey R, Karna SP. Atomically thin group V elemental films: theoretical investigations of antimonene allotropes. ACS Appl Mater Interface, 2015, 7: 11490-11496

49 Jones JE. On the determination of molecular fields. II. From the equation of state of a gas. Proc R Soc A-Math Phys Eng Sci, 1924, 106: $463-477$

50 Luo W, Xiang H. Room temperature quantum spin hall insulators with a buckled square lattice. Nano Lett, 2015, 15: 3230-3235

51 Ma Y, Dai Y, Kou L, et al. Robust two-dimensional topological insulators in methyl-functionalized bismuth, antimony, and lead bilayer films. Nano Lett, 2015, 15: 1083-1089

52 Bruzzone S, Fiori G. Ab-initio simulations of deformation potentials and electron mobility in chemically modified graphene and two-dimensional hexagonal boron-nitride. Appl Phys Lett, 2011, 99: 222108
53 Bardeen J, Shockley W. Deformation potentials and mobilities in non-polar crystals. Phys Rev, 1950, 80: 72-80

Acknowledgments This work was supported by the National Key Basic Research Program of China (2014CB931702), the National Natural Science Foundation of China (NSFC, 51572128), the NSFC and the Research Grants Council (RGC) of Hong Kong Joint Research Scheme (5151101197), and the Priority Academic Program Development of Jiangsu Higher Education Institutions.

Author contributions Cai B, Xie M, and Zeng $\mathrm{H}$ conceived the study. Cai B, Xie M, Zhang S, and Chen X performed the band structure and mobility calculations. Huang $\mathrm{C}$ and Kan $\mathrm{E}$ checked topological invariant Z2. Cai B, and Xie M analyzed the data and wrote the manuscript. Gu Y and Zeng $\mathrm{H}$ revised the manuscript. All authors read and approved the final manuscript.

Conflict of interest The authors declare that they have no conflict of interest.

Supplementary information Supplementary information is available in the online version of this article. 


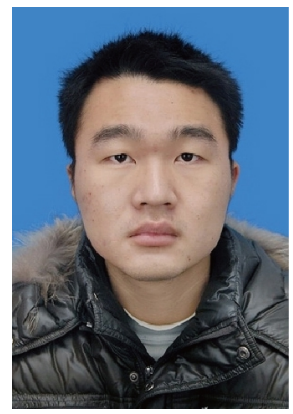

Bo Cai received his bachelor degree in material science and engineer from Nanjing University of Science and Technology in 2013. Now, he is a graduate student in Prof. Zeng's group. His current research interests include 2D materials simulation.

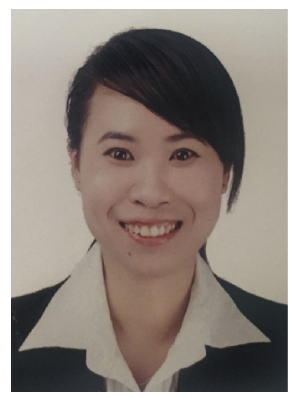

Meiqiu Xie received her master degree from Wuhan University of Technology in 2014. Now, she is a PhD candidate in Prof. Zeng's group in Nanjing University of Science and Technology. Her current research interests are concentrating on exploring the 2D material application in optoelectronics.

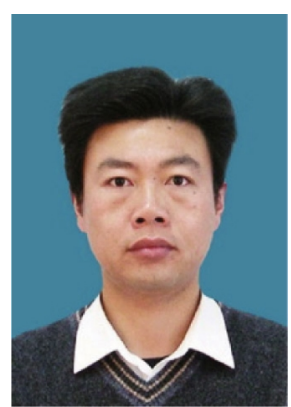

Haibo Zeng received his PhD degree from the Institute of Solid State Physics, Chinese Academy of Sciences in 2006. He later worked with Prof. Claus Klingshirn in 2007 at the University of Karlsruhe, Germany. In 2008, he joined Prof. Yoshio Bando's group at the National Institute for Materials Science (NIMS), Japan, under the support of Japan Society for the Promotion of Science (JSPS). In 2011, he returned to Nanjing University of Aeronautics and Astronautics as a full professor of materials science, and then moved to Nanjing University of Science and Technology in 2013 as a Distinguished Professor and Director of the Institute of Optoelectronics \& Nanomaterials. His current research interest is low-dimensional semiconductor optoelectronics, including 2D materials (calculation and experiment) and QDs (synthesis, optics, photodetectors and LEDs). So far, he has published over 150 peer-reviewed papers with more than 6,000 citations.

\section{二维沟道材料的潜力股：单层磷锑化合物}

蔡波 ${ }^{1 \dagger}$, 谢美秋 ${ }^{1 \dagger}$, 张胜利 $^{1}$, 黄呈熙 $^{2}$, 阙二军 ${ }^{2}$, 陈显平 ${ }^{3}$, 顾宇 ${ }^{1}$, 曾海波 $^{*}$

摘要 晶体管作为现代电子产业的基石, 要求其沟道材料既具有一个合适的带隙又具有较高的迁移率. 目前, 少层黑磷的结果显示, 黑磷既具 有一个适中的带隙值, 又拥有高于 $\mathrm{MoS}_{2}$ 的迁移率. 但是, 目前实验上黑磷迁移率的最大值仅达到 $1000 \mathrm{~cm}^{2} \mathrm{~V}^{-1} \mathrm{~s}^{-1}$ 左右, 与传统半导体 $\mathrm{Si}(1,400$ $\left.\mathrm{cm}^{2} \mathrm{~V}^{-1} \mathrm{~s}^{-1}\right)$ 和InP $\left(5,400 \mathrm{~cm}^{2} \mathrm{~V}^{-1} \mathrm{~s}^{-1}\right)$ 相比还是略逊一筹. 本文通过第一性原理模拟, 设计了一种新型二维半导体材料一单层 $S b P$. 结果表明, 单 层SbP可以稳定存在, 并且带隙值处在刚好合适的范围内, 同时, 迁移率可以高达 $20,000 \mathrm{~cm}^{2} \mathrm{~V}^{-1} \mathrm{~s}^{-1}$. 综合考虑到实验上同种相结构的AsP已经 被成功制备得到, 和理论上预测 $\mathrm{H}_{2}, \mathrm{O}_{2}$ 和 $\mathrm{H}_{2} \mathrm{O}$ 仅会通过不明显影响其性质的物理吸附吸附在锑烯表面, 我们设计的磷锑化合物会是一种具 备实验可能性、高度空气稳定性、并且对人体无毒的高性能二维沟道材料. 